\title{
Schematic Interpretation of Anomalies in the Physical Properties of Eu and $\mathrm{Yb}$ Among the Lanthanides
}

\author{
Yoshiharu Mae \\ Maetech, Mimuro, Midori Ward, Saitama City, Japan \\ Email address: \\ yoshimae@hotmail.com \\ To cite this article: \\ Yoshiharu Mae. Schematic Interpretation of Anomalies in the Physical Properties of Eu and Yb Among the Lanthanides. International \\ Journal of Materials Science and Applications. Vol. 6, No. 4, 2017, pp. 165-170. doi: 10.11648/j.jimsa.20170604.11
}

Received: May 24, 2017; Accepted: June 2, 2017; Published: June 19, 2017

\begin{abstract}
Lanthanides are the elements in $6^{\text {th }}$ period and the $3^{\text {rd }}$ group of the periodic table. Eu and $\mathrm{Yb}$ exhibit some unusual properties compared with the other lanthanides. The author has proposed a diagram to systematically illustrate the properties of the elements, by plotting the Young's modulus on the ordinate and thermal conductivity on the abscissa. Eu and Yb have much lower Young's moduli, and are located far from other lanthanides on the diagram. Most lanthanides have hexagonal structures. $\mathrm{Eu}$, however, has a body-centered cubic structure, because it is located on the extension of the curve of alkali metals. $\mathrm{Yb}$ has a face-centered cubic (fcc) structure, because it is located on the curve of fcc metals. The positions of Eu and $\mathrm{Yb}$ on the diagram are thought to act as a bridge between the lanthanides and other adjacent element groups. As a result, Eu and $\mathrm{Yb}$ work as if they were members in the adjacent element groups, which leads to their large atomic radii and their low melting points, etc.
\end{abstract}

Keywords: Eu, Yb, Lanthanides Crystal Structure, Atomic Radius, Melting Point

\section{Introduction}

It is known that $\mathrm{Eu}$ and $\mathrm{Yb}$ show unique properties in the crystal structure, atomic radius, melting temperature and so on, compared with the other lanthanides. This subject has been long deserted, but now the author has found the clue to it and reports the results here.

Most of the elements in the periodic table are metallic or semi-metallic. The periodic table is a useful tool for fundamentally understanding these elements, but it is difficult to use it to account for specific metallurgical phenomena. The author has proposed to graphically represent metallic and semi-metallic elements, to enable their nature to be interpreted in more detail [1]. The main characteristics of metallic elements are their crystal structures and presence of free electrons. The Young's modulus and thermal conductivity are good indicators of the lattice strength and characteristics of free electrons, respectively. Each element is plotted on a diagram with thermal conductivity on the abscissa and Young's modulus on the ordinate. This TC (thermal conductivity)-YM (Young's modulus) diagram is hereafter referred to as "the diagram".

\section{Crystal Structures of Elements}

Metallic elements have specific crystal structures. The crystal structures of each element can be classified as body-centered cubic (bcc), face-centered cubic (fcc), hexagonal close-packed (hcp), diamond structures, and other miscellaneous structures [2]. The distribution of the crystal structures of the elements are shown on the diagram in Figure 1.

\section{a. Distribution of bec metals}

Elements with bcc structures largely lie on a straight line connecting $\mathrm{V}, \mathrm{Ta}, \mathrm{Cr}$, Mo and $\mathrm{W}$, which can be called the straight line of refractory metals. Fe is located near the straight line of refractory metals. On the other hand, alkali metals with bce structures lie on a curve near the abscissa, which can be called the curve of alkali metals.

b. Distribution of fec metals

Metals with fcc structures lie on a clear curve which can be called the curve of $f c c$ metals. All metals with fcc structures lie on this one line, and are distributed from low to high Young's modulus and from low to high thermal conductivity. Elements with both high Young's modulus and high thermal conductivity do not exist. 
c. Distribution of hcp metals and other miscellaneous structures

Elements with hcp and other miscellaneous structures are distributed elsewhere. The lanthanides mostly with hcp structures gather tightly in the low Young's modulus and low thermal conductivity region.

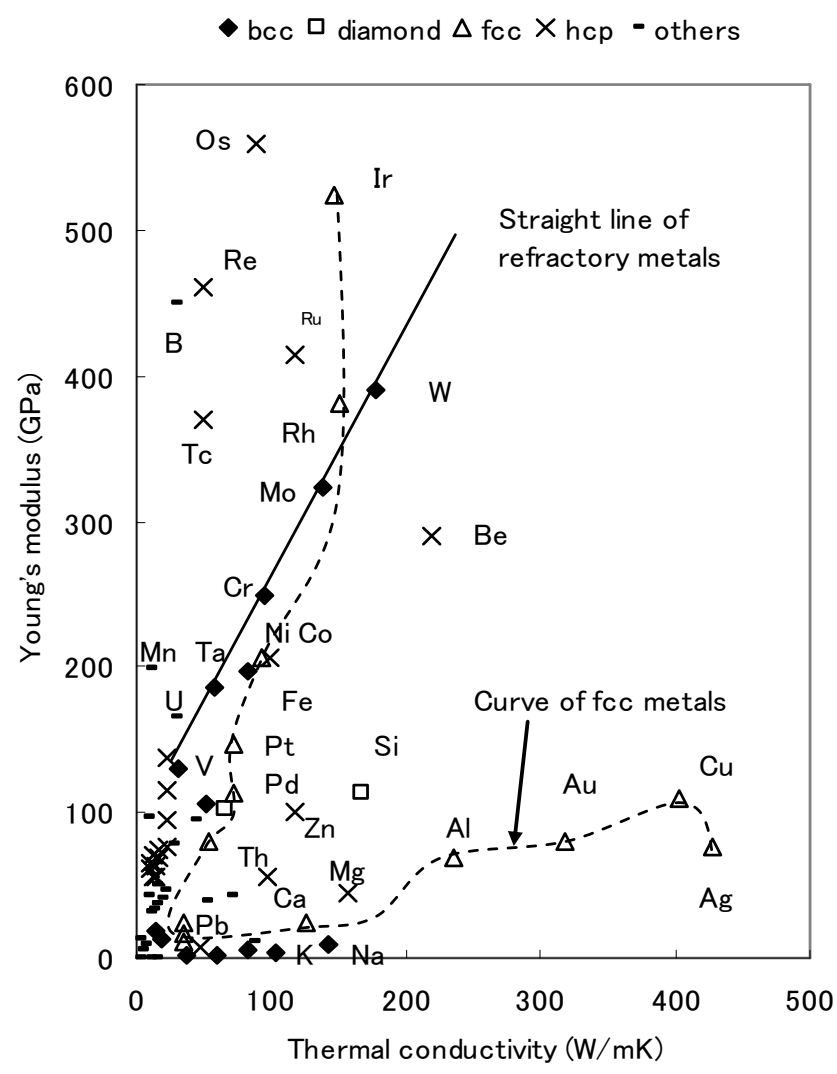

Figure 1. Crystal structures of elements shown on the diagram.

\section{Peculiar Properties of Eu and Yb}

The elements from ${ }^{57} \mathrm{La}$ to ${ }^{71} \mathrm{Lu}$ are known as the lanthanides. They exhibit similar chemical properties because they possess the same outer shell structure electrons, but some anomalies are observed in some elements such as $\mathrm{Eu}$ and $\mathrm{Yb}$. Eu and $\mathrm{Yb}$ are introduced in the chemical dictionary, as the elements with peculiar properties compared with other lanthanides [3].

\subsection{Crystal Structure}

Figure 2 shows the crystal structures of the lanthanides [4]. Most of them adopt hexagonal structures. Elements from ${ }^{57} \mathrm{La}$ to ${ }^{61} \mathrm{Pm}$ adopt double hexagonal structures. At ${ }^{62} \mathrm{Sm}$, the structure changes to rhombohedral. ${ }^{63} \mathrm{Eu}$ has a bcc structure. Elements from ${ }^{64} \mathrm{Gd}$ to ${ }^{69} \mathrm{Tm}$ have hep structures. ${ }^{70} \mathrm{Yb}$ suddenly adopts a fec structure, and ${ }^{71} \mathrm{Lu}$ adopts a hep structure again. In short, the crystal structures of Sm, Eu and $\mathrm{Yb}$ are exceptional among the lanthanides.

\section{$\Delta$ hcp $\square$ double hexagonal $\bigcirc$ rhombohedral $\boldsymbol{\nabla c c} \bullet$ fcc}

Atomic No.

55

60

65

70

75

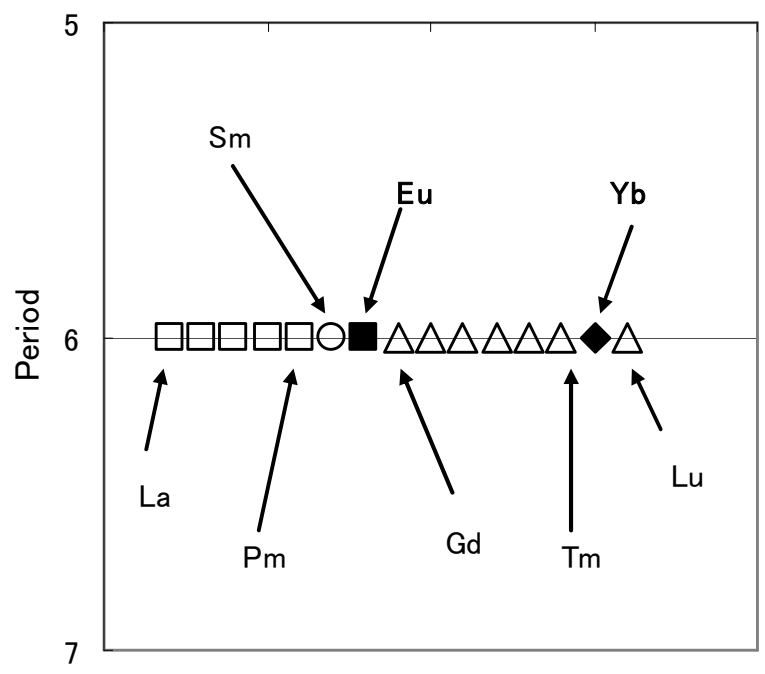

Figure 2. Crystal structures of lanthanides with atomic number.

\subsection{Atomic Radius}

Anomalies of $\mathrm{Eu}$ and $\mathrm{Yb}$ are also observed in their atomic radii. Figure 3 shows the variation of atomic radius of the lanthanides with atomic number. The atomic radius tends to decrease with increasing atomic number, which is known as the lanthanide contraction [4]. However, Eu and $\mathrm{Yb}$ show abrupt increases in atomic radius.

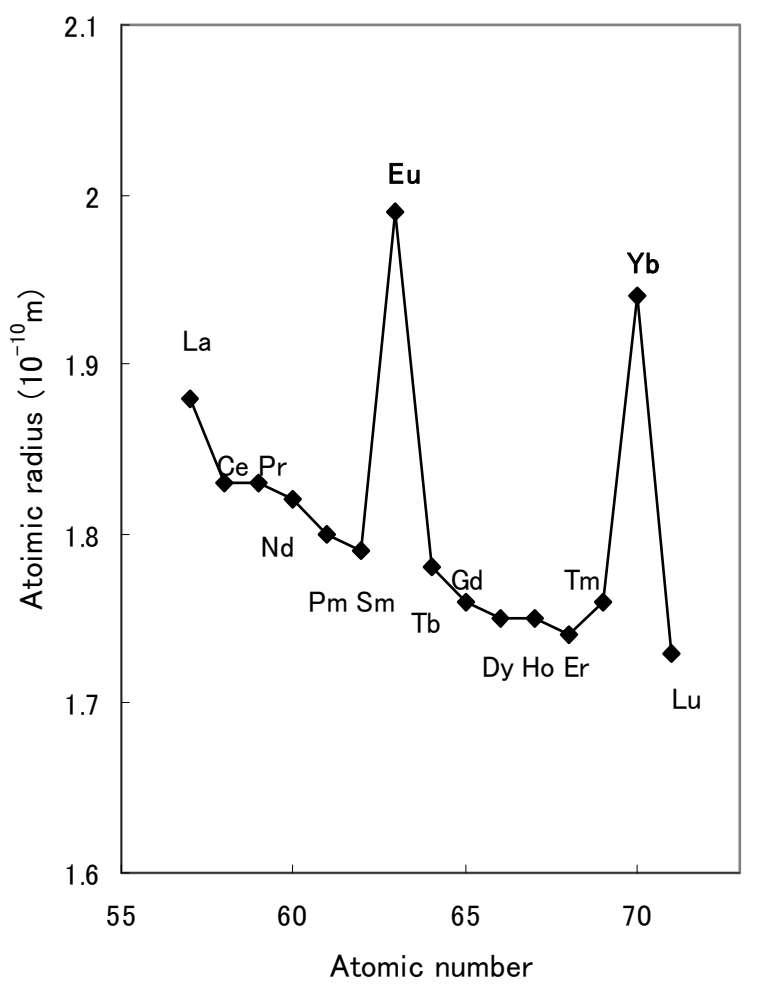

Figure 3. Atomic radii of lanthanides with atomic number. 
The Wigner-Seitz cell radii also show the same pattern and abrupt rises at $\mathrm{Eu}$ and $\mathrm{Yb}$ [5]. Gschneidner noted that $\mathrm{Eu}$ and $\mathrm{Yb}$ exhibited divalent characteristics while other lanthanides exhibited trivalent characteristics, and this was relevant to the increased atomic radii of $\mathrm{Eu}$ and $\mathrm{Yb}$ [6].

\subsection{Melting Temperature}

Figure 4 shows the variation of melting temperature of the lanthanides with atomic number. The melting temperature tends to increase with increasing atomic number, but abruptly decreases at Eu and $\mathrm{Yb}$.

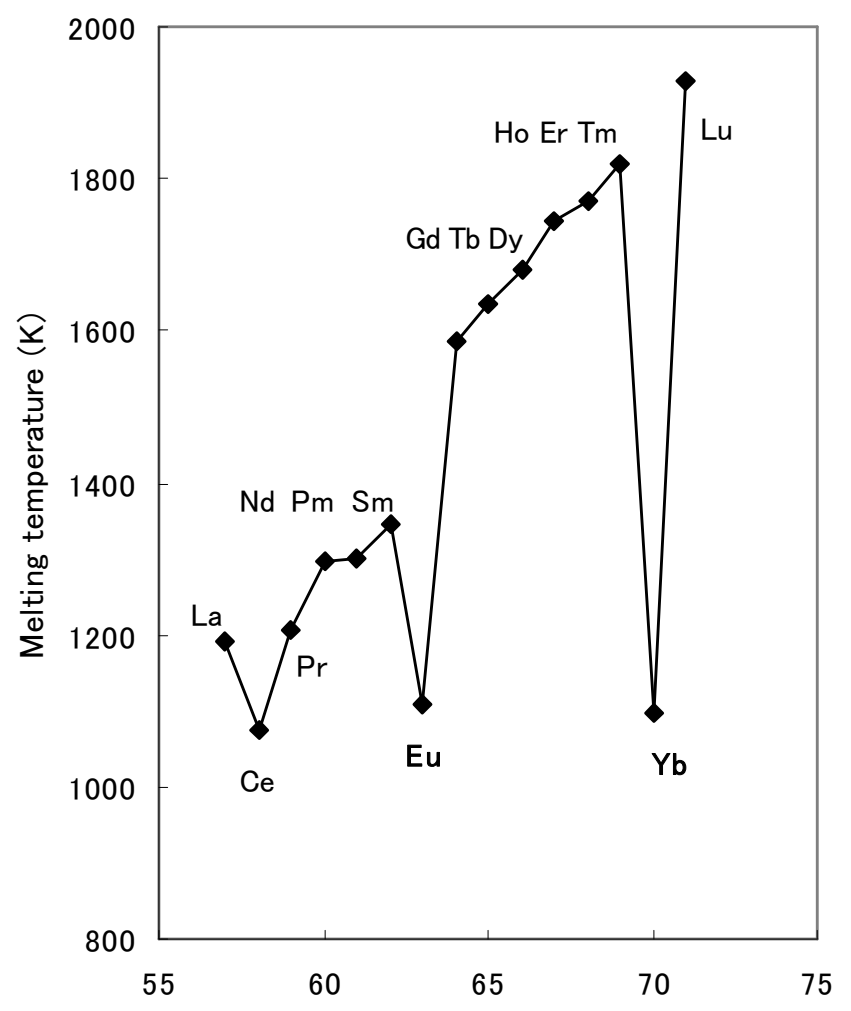

Atomic No.

Figure 4. Melting temperatures of lanthanides with atomic number.

Other properties such as thermal expansion coefficient, boiling point, heat of fusion and heat capacity exhibit similar trends. Such anomalies of $\mathrm{Eu}$ and $\mathrm{Yb}$ among the lanthanides were early recognized and somewhat accounted for by valence electrons [6].

\section{Representation of $\mathrm{Eu}$ and $\mathrm{Yb}$ on the Diagram}

\subsection{Crystal Structure}

Figure 5 shows the distribution of crystal structures of the elements in the low Young's modulus and low thermal conductivity region of the diagram.

$\begin{array}{ll}\Delta \text { bcc } & \Delta \text { fcc } \\ \Delta \text { hcp } & \square \text { double hexagonal } \\ \Delta \text { hexagonal A8 } & \text { * monoclinic } \\ \text { X orthorhombic } & \text { O rhombohedral } \\ \text { - teragonal } & \square \text { others }\end{array}$

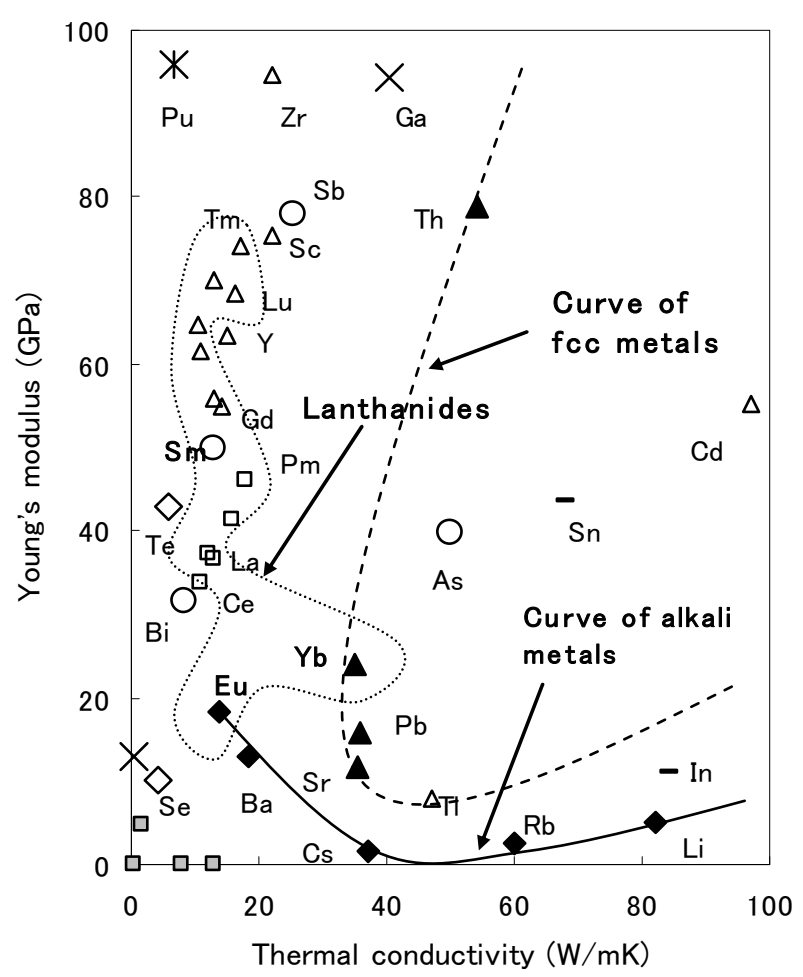

Figure 5. Crystal structures of elements in the low Young's modulus and low thermal conductivity region of the diagram.

The lanthanides are distributed over a wide Young's modulus range at mostly constant low thermal conductivity. The lower Young's modulus elements such as ${ }^{57} \mathrm{La},{ }^{58} \mathrm{Ce},{ }^{59} \mathrm{Pr}$, ${ }^{60} \mathrm{Nd}$ and ${ }^{61} \mathrm{Pm}$ adopt double hexagonal structure. The higher Young's modulus elements such as ${ }^{64} \mathrm{Gd},{ }^{65} \mathrm{~Tb},{ }^{66} \mathrm{Dy},{ }^{67} \mathrm{Ho}$, ${ }^{68} \mathrm{Er},{ }^{69} \mathrm{Tm}$ and ${ }^{71} \mathrm{Lu}$ adopt hep structure. ${ }^{62} \mathrm{Sm}$ is a transient element and lying between these groups, adopting a rhombohedral structure.

${ }^{63} \mathrm{Eu}$ and ${ }^{70} \mathrm{Yb}$ do not follow this trend, and are located surprisingly at much lower Young's modulus positions.

Eu is located at lower Young's modulus and lower thermal conductivity, and lies on the extension of the curve of alkali metals. The curve of alkali metals contains $\mathrm{Na}, \mathrm{K}, \mathrm{Li}, \mathrm{Rb}$ and $\mathrm{Cs}$, ranging from high to low thermal conductivity. The alkali earth metal $\mathrm{Ba}$ also lies on the extension of the curve of alkali metals. Eu lies on this extension line after $\mathrm{Ba}$. This is the reason why $\mathrm{Eu}$ adopts a bcc structure, and does not follow the trend of the other lanthanides.

$\mathrm{Yb}$ is located at low Young's modulus but slightly higher thermal conductivity than $\mathrm{Eu}$. It is located near $\mathrm{Pb}$, which lies on the curve of fcc metals. The curve of fcc metals proceeds from $\mathrm{Ag}$ (far right side) through $\mathrm{Cu}, \mathrm{Au}, \mathrm{Al}, \mathrm{Ca}, \mathrm{Pb}, \mathrm{Th}, \mathrm{Pd}$, $\mathrm{Pt}, \mathrm{Ni}$, and $\mathrm{Rh}$, and ends at $\mathrm{Ir}$, as shown in Figure 1. $\mathrm{Yb}$ and $\mathrm{Sr}$ are located near $\mathrm{Pb}$. This is the reason why $\mathrm{Yb}$ adopts a fcc structure and does not follow the trend of the lanthanides. 
This is also the reason why Sr adopts a fcc structure, different from other alkali earth metals.

\subsection{Atomic Radius}

Figure 6 shows the distribution of atomic radii of elements of low Young's modulus and low thermal conductivity.

$\bullet<1.2 \square 1.2-1.4 \Delta 1.4-1.5 \Delta 1.5-1.6 \square 1.6-1.7$

$\diamond 1.7-1.8 \times 1.8-1.9 * 1.9-2.0+2.0-2.2 \circ 2.2-2.6$

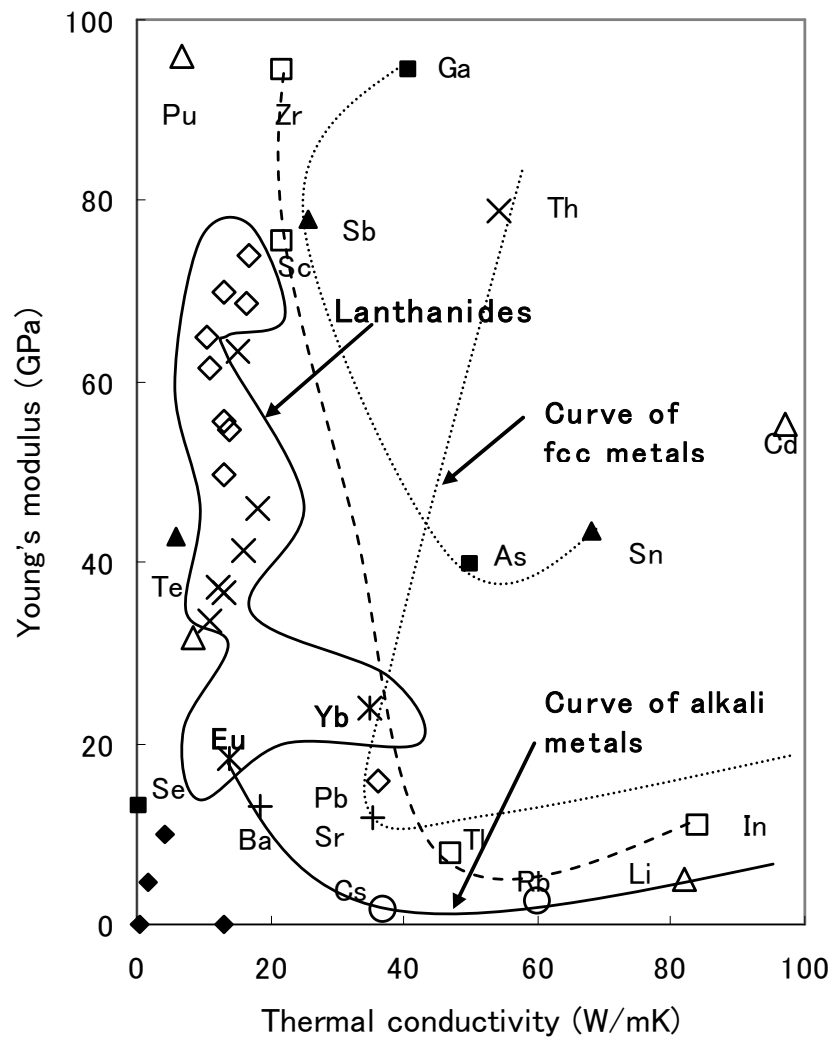

Figure 6. Atomic radii of elements in the low Young's modulus and low thermal conductivity region of the diagram.

The figures in the legend show the range of atomic radii in units of $1 \times 10^{-10} \mathrm{~m}$. Ga, Sb, As and $\mathrm{Sn}$ are located in an arch near the center of the diagram and have the smallest atomic radii. $\mathrm{Zr}, \mathrm{Sc}, \mathrm{Tl}$ and $\mathrm{In}$ form an outer concentric circle and have the next smallest atomic radii. The atomic radius increases with decreasing Young's modulus and decreasing thermal conductivity. The lanthanides are located outside of these central elements, and have much larger atomic radii. As to the lanthanides, atomic radius increases with decreasing Young's modulus. This means that $\mathrm{Eu}$ and $\mathrm{Yb}$ have large atomic radii. $\mathrm{Eu}$ connects to $\mathrm{Ba}$, and $\mathrm{Yb}$ connects to $\mathrm{Pb}$ and $\mathrm{Sr}$, respectively. This is the reason why the atomic radii of $\mathrm{Eu}$ and $\mathrm{Yb}$ abruptly increase with increasing atomic number, as shown in Figure 3.

\subsection{Melting Temperature}

Figure 7 shows the distribution of melting temperatures (K) of elements with low Young's modulus and low thermal conductivity.

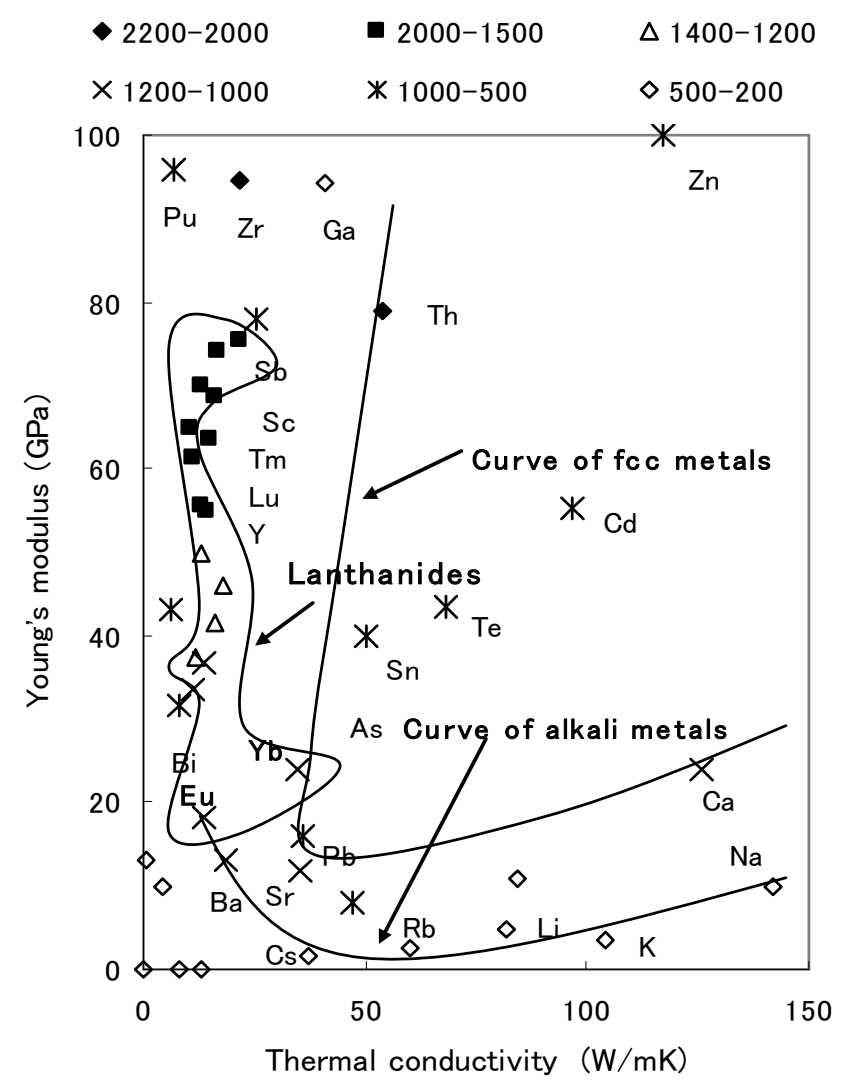

Figure 7. Melting temperatures (K) of elements in the low Young's modulus and low thermal conductivity region of the diagram.

Among the lanthanides, the melting temperature decreases with decreasing Young's modulus. Eu and Yb occupy the lowest positions of the lanthanides and have the lowest melting temperatures. Eu connects smoothly to $\mathrm{Ba}$, which has a similar melting temperature. $\mathrm{Yb}$ connects smoothly to $\mathrm{Sr}$, which has a similar melting temperature. This is the reason why the melting temperatures of $\mathrm{Eu}$ and $\mathrm{Yb}$ abruptly decrease with increasing atomic number, as is shown in Figure 4.

\subsection{Valence}

It is reported by Gschneidner and other authors that Eu and $\mathrm{Yb}$ exhibit divalent characteristics while other lanthanides exhibit trivalent characteristics [6] [7]. Gschneidner showed this behavior as shown in Figure 8.

This is essentially the same figure as Figure 3, except that the valence characteristics are added. $\mathrm{Eu}$ and $\mathrm{Yb}$ adopt divalence, while most of the lanthanides adopt trivalence. He referred their large atomic radii as the cause of the divalence. But their large atomic radii and divalence are equally the results of their low Young's moduli.

This can be explained from their locations on the diagram. $\mathrm{Eu}$ is located close to Ba lying on the same curve of alkali metals on the diagram, therefore, $\mathrm{Eu}$ shows the similar behavior as $\mathrm{Ba}$ in the valence. $\mathrm{Yb}$ is located close to Sr lying on the same curve of fcc metals, therefore, $\mathrm{Yb}$ shows the similar behavior as $\mathrm{Sr}$ in the valence. As a result, both Eu and $\mathrm{Yb}$ show divalence, while other lanthanides show trivalence. 
Gschneidner was right in the point that he related $\mathrm{Eu}$ and $\mathrm{Yb}$ to $\mathrm{Ba}$, as shown in Figure 8.

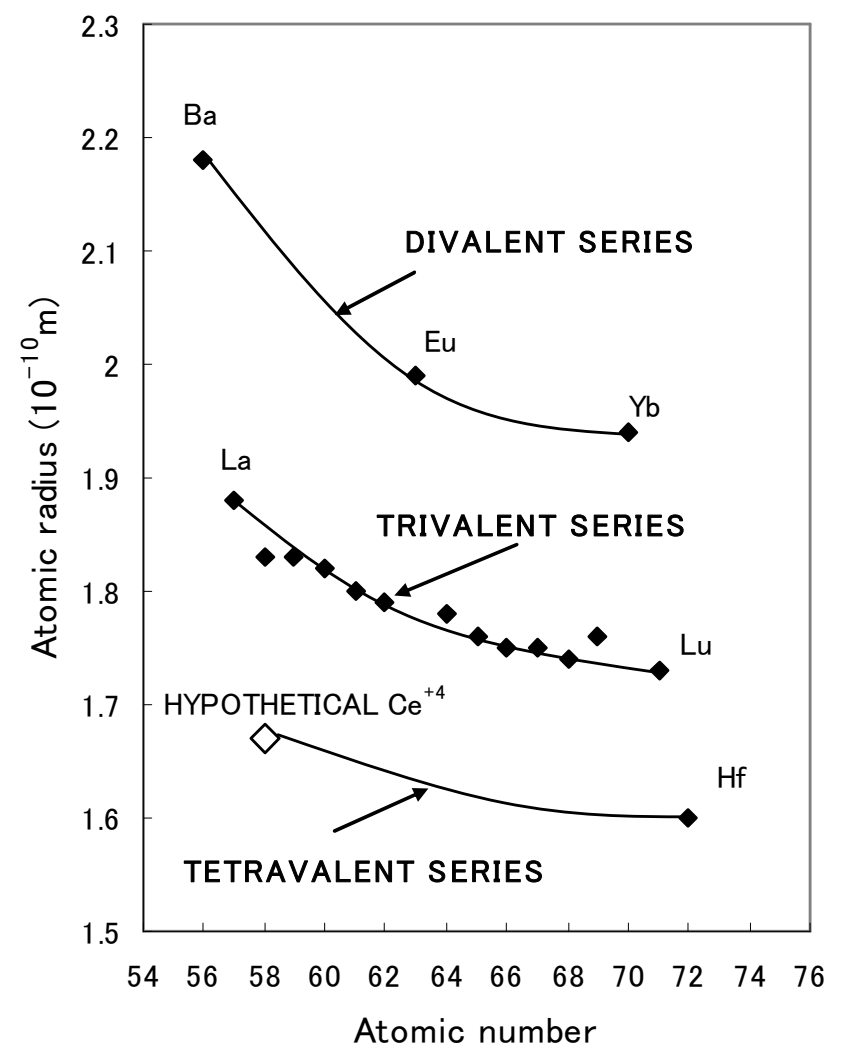

Figure 8. The relationship between atomic radii, valence and atomic number by Gshneidner.

\section{Discussion}

Anomalies of $\mathrm{Eu}$ and $\mathrm{Yb}$ in the crystal structures, atomic radii, melting temperatures and valences are observed with increasing atomic number. Similar anomalies are observed to a greater or lesser degree in the thermal expansion, boiling point, heat of fusion, and vapor pressure, etc. As mentioned above, the properties of $\mathrm{Eu}$ and $\mathrm{Yb}$ are strongly related to their positions in the lower Young's modulus region of the diagram. Why are $\mathrm{Eu}$ and $\mathrm{Yb}$ located there? They seem to play a role in bridging the lanthanides to other element groups. Such bridging effect can be seen elsewhere. $\mathrm{Nb}$ is a member of the refractory metals, but lies off the straight line of refractory metals, and is located near Ge as shown in Figure 1. $\mathrm{Nb}$ is bcc-structured, and Ge is diamond-structured. They are non-closed-packed. $\mathrm{Nb}$ seems to play a role bridging the refractory metals group to the diamond structure group of $\mathrm{Ge}$ and $\mathrm{Si}$. Similarly, $\mathrm{Eu}$ and $\mathrm{Yb}$ connect to the alkali metals and fcc metals, respectively. As a result, Eu adopts a bcc structure, and $\mathrm{Yb}$ a fcc structure.

The unique physical properties of $\mathrm{Eu}$ and $\mathrm{Yb}$ such as large atomic radii and low melting temperatures stem from their low Young's moduli. What's the mechanism of their low Young's moduli? Figure 9 shows the electronic configurations of the lanthanides [8].

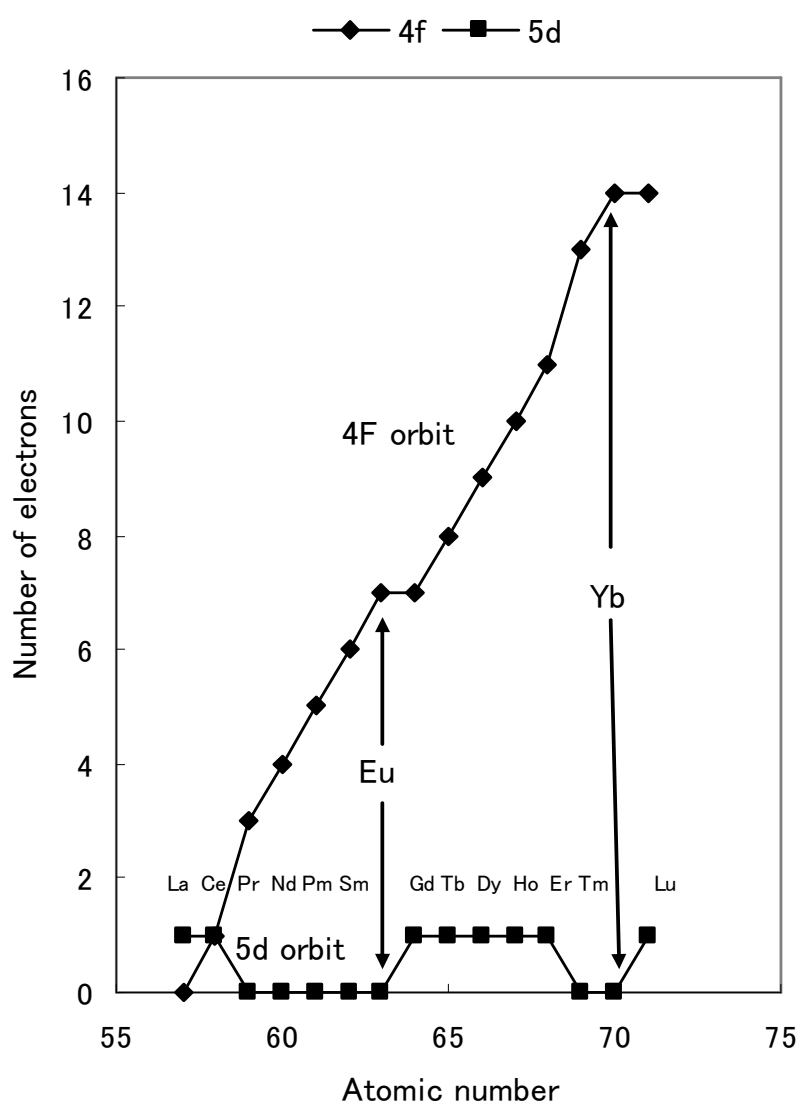

Figure 9. Electronic configurations of lanthanides.

The electrons progressively fill the $4 \mathrm{f}$ orbital, and the electron occupancy of the $5 \mathrm{~d}$ orbital varies between zero to one. $\mathrm{Eu}$ and $\mathrm{Yb}$ are the last elements where the $5 \mathrm{~d}$ orbital is empty.

The Young's modulus is generally thought to be an engineering parameter, but it is a physically fundamental factor. The Young's modulus is proportional to the gradient of the tangent of the curve of the Condon-Morse force between atoms at an equivalent atomic distance. Thus, the Young's modulus is proportional to the second order differential of the binding potential between two atoms at equivalent atomic distance [9]. Therefore, the Young's modulus is a good index to represent the binding state of atoms.

The low Young's modulus of $\mathrm{Yb}$ is said to stem from the closed-shell electron configuration with the full $4 \mathrm{f}$ orbit, thus only the two 6s electrons work for metallic bonding in $\mathrm{Yb}$ [10]. It leads to its lower Young's modulus. In Eu, the $4 \mathrm{f}$ orbit is half occupied. It seems to lead to the low Young's modulus of Eu also.

In this way, $\mathrm{Eu}$ and $\mathrm{Yb}$ become to occupy the low Young's modulus region in the TC-YM diagram, and connect to the adjacent other element groups such as the alkali metals and the fcc metals. As a result, they behave like the members of the adjacent element groups. This is the reason why Eu and $\mathrm{Yb}$ show the anomalies in the physical properties among the lanthanides. 


\section{Conclusion}

The unique electron configurations of Eu and $\mathrm{Yb}$ cause their low Young's moduli. Their low Young's moduli are the source of their peculiar properties.

They connect to the adjacent other element groups on the diagram. They behave like the members of the adjacent element groups. Eu lies on the curve of alkali metals, and $\mathrm{Yb}$ lies on the curve of fcc metals. As a result, Eu adopts bcc structure, and $\mathrm{Yb}$ adopts fcc structure, respectively. They have also large atomic radii, low melting temperatures, divalence and so on, affected by their adjacent elements groups.

\section{References}

[1] Y. Mae, "What the Darken-Gurry plot means about the solubility of elements in metals," Metall. Mater. Trans. A, vol. 47, pp. 6498-6506, Dec., 2016.

[2] Japan Institute of Metals, Metals data book, $3^{\text {rd }}$ ed., Maruzen, Tokyo, 1993, pp. 36-43.
[3] Tokyo-kagaku-doujin, Big chemical dictionary, Tokyo-kagaku-doujin, Tokyo, 1989, p. 190.

[4] K. N. R. Taylor, M. L. Darby, Physics of rare earth solids, Chapman and Hall, London, 1972, pp. 60-62.

[5] J. Bernstein, Plutonium, Sangyo-tosho, Tokyo, 2008, p. 174.

[6] E. C. Subbarao and W. E. Wallace, Science and technology of rare earth materials, Academic Press, New York, 1980, pp. 53-54.

[7] R. A. Cooley, D. M. Yost, H. W. Stone, "Europium( II ) salts", Inorganic Synthesis, 2, pp69-73, 1946.

[8] Japan Institute of Metals, Metals data book, $3^{\text {rd }}$ ed., Maruzen, Tokyo, 1993, p. 5.

[9] W. Hayden, W. G. Moffat, and J, Wulff, The structure and properties of materials, Vol. III Mechanical behavior, John Wiley and Sons, New York, 1965, pp. 26-31.

[10] A. F. Holleman, E. Wiberg, N. Wiberg, Die Lanthanoide, Walter de Gruyter, Belrin, 1985, pp. 1265-1279. 